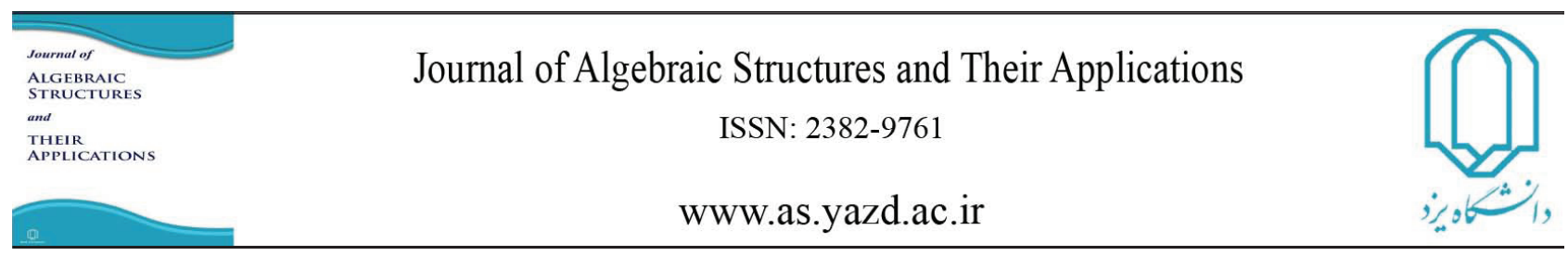

Algebraic Structures and Their Applications Vol. 4 No. 1 ( 2017 ) pp 33-42.

\title{
ON THE EDGE-DIFFERENCE AND EDGE-SUM CHROMATIC SUM OF THE SIMPLE GRAPHS
}

\author{
S. RAHIMI SHARBAF AND KH. ERFANI*
}

Communicated by A.R. Ashrafi

\begin{abstract}
For a coloring $c$ of a graph $G$, the edge-difference coloring sum and edge-sum coloring sum with respect to the coloring $c$ are respectively $\sum_{c} D(G)=\sum|c(a)-c(b)|$ and $\sum_{s} S(G)=\sum(c(a)+c(b))$, where the summations are taken over all edges $a b \in E(G)$. The edge-difference chromatic sum, denoted by $\sum D(G)$, and the edge-sum chromatic sum, denoted by $\sum S(G)$, are respectively the minimum possible values of $\sum_{c} D(G)$ and $\sum_{c} S(G)$, where the minimums are taken over all proper coloring of $c$. In this work, we study the edge-difference chromatic sum and the edge-sum chromatic sum of graphs. In this regard, we present some necessary conditions for the existence of homomorphism between two graphs. Moreover, some upper and lower bounds for these parameters in terms of the fractional chromatic number are introduced as well.
\end{abstract}

\section{INTRODUCTION}

The general graph proper-coloring is one of the most well-known problems in combinatorial optimization. Besides its theoretical significance as a canonical NP-Hard problem [4], graph http://dx.doi.org/10.29252/asta.4.1.33

MSC(2010): Primary:05C30, 05C15

Keywords: edge-difference chromatic sum, edge-sum chromatic sum, graph homomorphism, Kneser graph, fractional chromatic number.

Received: 23 Nov 2017, Accepted: 23 Jan 2018.

*Corresponding author

(C) 2017 Yazd University. 
coloring arises naturally in a variety of real-world applications such as timetable problems[[]], warehouse management[5], frequency allocation in mobile network[8], register allocation in optimizing compilers[9], scheduling problem[[10], design and operation of flexible manufacturing systems[II]].

Vertex sum coloring problem is a special case of general graph coloring in which main aim is to minimize the sum of colors, where the colors are represented by natural numbers. It is closely related to the basic graph coloring problem and it was introduced by Kubicka [2] for the first time. It is known to be NP-hard with several practical applications including VLSI design, scheduling, and distributed resource allocation (see [2T] for a list of references) and also has been investigated in literature [12, 14, 15, 17, 18, 1.9].

Let $G=(V, E)$ be a simple graph. A proper vertex-coloring of $G$ is an assignment $c: V \rightarrow \mathbb{N}$ such that each of two adjacent vertices are received different colors. The vertex chromatic sum of $G$ with respect to the proper coloring $c$ is $\sum_{c}(G)=\sum_{v \in V(G)} c(v)$. The vertex chromatic sum of $G$, denoted by $\sum G$, is defined to be $\min _{c} \sum_{v \in V(G)} c(v)$, where the minimum is taken aver all proper-colorings of $G$. The vertex strength of $G$, denoted by $s(G)$, is the smallest number $s$ such that there is a proper coloring $c: V(G) \longrightarrow\{1, \ldots, s\}$ for which $\sum_{c}(G)=\sum G$.

Let $G$ and $H$ be two graphs. A homomorphism from $G$ to $H$ is a map $f: V(G) \longrightarrow V(H)$ which preserves adjacency, that is, if $u v \in E(G)$, then $f(u) f(v) \in E(H)$. Many classical problems in combinatorics can be redefined in terms of homomorphism. For instance, the chromatic number of a graph $G$, denoted by $\chi(G)$, is the minimum possible value of $n$ for which there is a homomorphism from $G$ to the complete graph $K_{n}$. Also, the fractional chromatic number of $G$, denoted by $\chi_{f}(G)$, is the infimum of the ratio $\frac{m}{n}$ such that there is a homomorphism from $G$ to the Kneser graph $K G(m, n)$. It is known that the infimum can be replaced by minimum, see [22].

In [T], Alishahi and Taherkhani obtained a necessary condition for the existence of homomorphism between two graphs based on their chromatic sums. They used this result to obtain an upper bound for the chromatic sum of a graph $G$ in terms of its fractional chromatic number $\left(|V(G)| \chi_{f}(G)>\sum G\right)$. In this paper, we introduce some other necessary conditions for the existence of homomorphism between graphs in terms of their edge-difference chromatic sum and edge-sum chromatic sum. Using these results, we introduce some upper bounds for the edge-difference chromatic sum and edge-sum chromatic sum of graphs based on their fractional chromatic numbers.

The rest of this paper is organized as follows. In the first section, we will introduce edgedifference chromatic sum, denoted by $\sum D(G)$, and the edge sum chromatic sum, denoted by $\sum S(G)$. Next section is devoted to introduce Kneser graphs and find some upper bounds for edge-difference chromatic sum and edge-sum chromatic sum of Kneser graphs. The necessary 
conditions for the existence of homomorphism between two graphs will be studied in Section 3 . Finally, two lower bounds for fractional chromatic number of graph will be introduced in Section4.

\section{The EDGE-DIFFERENCE AND EDGE-SUM CHROMATIC SUM}

Definition 2.1. Let $c: V \rightarrow I$ be a proper coloring of $G$. The edge-difference sum coloring and edge-sum sum coloring related to $c$, are indicated by $\sum_{c} D(G)$ and $\sum_{c} S(G)$, and defined as follows, respectively

$$
\begin{aligned}
& \sum_{c} D(G)=\sum_{(u, v) \in E(G)}|c(u)-c(v)|, \\
& \sum_{c} S(G)=\sum_{(u, v) \in E(G)}(c(u)+c(v)) .
\end{aligned}
$$

Definition 2.2. Let $C$ be the set of all proper coloring of $G$. The edge-difference chromatic sum and edge-sum chromatic sum, are denoted by $\sum D(G)$ and $\sum S(G)$ and defined as follows, respectively

$$
\begin{aligned}
& \sum D(G)=\min \left\{\sum_{c} D(G) \mid c \in C\right\}, \\
& \sum S(G)=\min \left\{\sum_{c} S(G) \mid c \in C\right\}
\end{aligned}
$$

and the smallest number of color $c$ for $\sum_{c} D(G)=\sum D(G)$ and $\sum_{c} S(G)=\sum S(G)$ are denoted by $d s(G)$ and $s s(G)$, respectively.

Trivially, $\chi(G) \leq d s(G)$, and sometimes inequality strictly holds (see Figure (U)), and $s s(G) \neq s(G)$ in general (see Figure (『)).

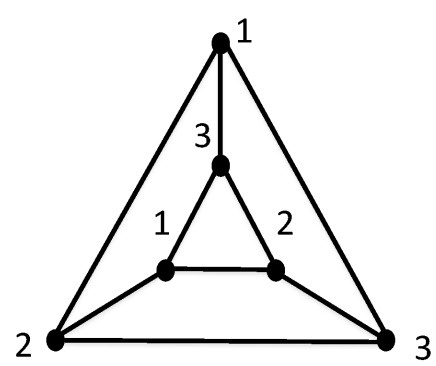

(A) $\chi(G)=s s(G)=3$

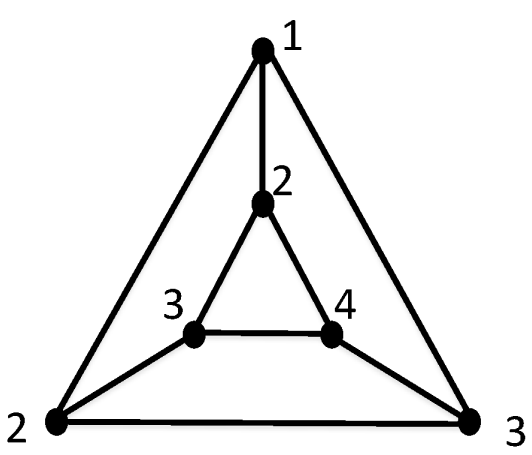

(в) $d s(G)=4, \sum D(G)=11$

FiguRE 1. The difference chromatic sum requires 4 colors. 


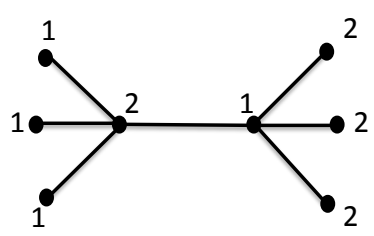

(A) $s s(G)=2$

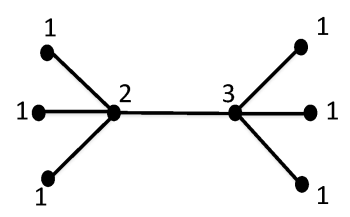

(B) $s(G)=3$

FiguRE 2. The sum chromatic sum and the vertex chromatic sum .

Assume that $f$ is a proper coloring for vertices of $G$ and $d(v)$ denotes the degree of vertex $v$ of $G$. Moreover,

$$
\begin{aligned}
& \sum_{(u, v) \in E(G)}(f(u)+f(v))=\sum_{v \in V(G)} d(u) f(u) \\
\Rightarrow & \delta \sum_{v \in V(G)} f(u) \leq \sum_{(u, v) \in E(G)}(f(u)+f(v)) \leq \Delta \sum_{v \in V(G)} f(u),
\end{aligned}
$$

where $\Delta$ and $\delta$ are maximum and minimum degrees in graph $G$, repectively. Therefore it's trivial that

$$
\begin{aligned}
& \delta \min \left\{\sum_{v \in V(G)} f(u)\right\} \leq \min \left\{\sum_{(u, v) \in E(G)}(f(u)+f(v))\right\} \leq \Delta \min \left\{\sum_{v \in V(G)} f(u)\right\} \\
& \Rightarrow \delta \sum G \leq \sum S(G) \leq \Delta \sum G .
\end{aligned}
$$

In fact the minimization in inequality (6) is on any proper coloring $f$. So, we can obtain some upper and lower bound for $\sum G$ based on $\sum S(G)$.

$$
\left\lceil\frac{\sum S(G)}{\Delta}\right\rceil \leq \sum G \leq\left\lfloor\frac{\sum S(G)}{\delta}\right\rfloor
$$

It should be noted that the latter bound more tightly, if the values of $\Delta$ and $\delta$ are close to

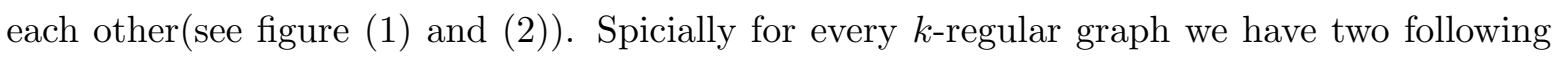
Propositions.

Proposition 2.3. For every k-regular graph $G$ we have $\sum S(G)=k \sum G$ and $s s(G)=s(G)$.

Proposition 2.4. For every k-regular graph $G$, we have $\frac{\sum G}{\sum S(G)}=\frac{|V(G)|}{2|E(G)|}$.

For example for Petersen graph $\sum(P)=19$ and therefore $\sum S(G)=3 \times 19=57$.

\section{The Upper Bounds For $\sum D(K G(m, n)), \sum S(K G(m, n))$}

Let $m$ and $n$ be two positive integers. We denote by $[m]$ the set $\{1,2, \ldots, m\}$, and denote by $\left(\begin{array}{c}{[m]} \\ n\end{array}\right)$ the collection of all $n$-subsets of $[m]$. The Kneser graph with parameters $m$ and $n$ with $m \geq 2 n$, denoted by $K G(m, n)$, is the graph with vertex set $\left(\begin{array}{c}{[m]} \\ n\end{array}\right)$ such that two vertices 
are adjacent if and only if the corresponding subsets are disjoint. For $i=1,2, \ldots, m-n+1$, define $A_{i}$ to be the set of all $n$-subsets of $[m]$ containing $i$ and having no intersection with $\{1,2, \ldots, i-1\}$.

In this section, we introduce an upper bound for $\sum D(K G(m, n))$ and $\sum S(K G(m, n))$. It should be noted that to avoid redundancy, details of calculation are not shown. But all of them can be confirmed using Maple or Matlab.

Proposition 3.1. Let $m$ and $n$ be two positive integers, where $m \geq 2 n$.

a) For $i=1,2, \ldots, m-n+1$, the set $A_{i}$ is an independent set of $K G(m, n)$.

b) The sets $A_{i}$ partition the vertex set of $K G(m, n)$.

Proof. Since each element in $A_{i}$ contains $i$, it is clear that $A_{i}$ is an independent set of $K G(m, n)$. Now, note that for each $v \in V(K G(m, n))$, we have $v \in A_{i}$, where $i=\min v$, which implies that the union of $A_{i}$ 's is $V(K G(m, n))$. To complete the proof, we must show that $A_{i}$ 's are pairwise disjoint. For a contradiction, assume that there are $i \neq j \in[m-n+1]$, such that $A_{i} \cap A_{j} \neq \varnothing$. Consider $v \in A_{i} \cap A_{j}$. Note that we must have $\min v=i$ if and only if $v \in A_{i}$. This observation implies $i=j=\min v$, which is a contradiction.

Note that $\left|A_{i}\right|=\left(\begin{array}{c}m-i \\ n-1\end{array}\right)$ and $\left|A_{i+1}\right|<\left|A_{i}\right|$ for $i=1,2, \ldots, m-n+1$. Furthermore, one can check that there is no edges between vertices in $\bigcup_{i=m-2 n+2}^{m-n+1} A_{i}$. Therefore

$$
A_{1}, A_{2}, \ldots, A_{m-2 n+1}, B_{m-2 n+2}
$$

are independent sets, where

$$
B_{m-2 n+2}=\bigcup_{i=m-2 n+2}^{m-n+1} A_{i} .
$$

Let $G$ be a graph and $S$ be an arbitrary independent set of $G$. As an immediate consequence of the definition of the edge-difference chromatic sum, we have

$$
\sum D(G) \leq \sum_{c^{\prime}} D(G)=\sum_{(u, v) \in I}\left|c^{\prime}(u)-c^{\prime}(v)\right|+\sum D(G \backslash S),
$$

where $I$ is the set of all edges between $S$ and $G \backslash S$ (see Figure (B)) and

$$
c^{\prime}(v)= \begin{cases}\min \{c(u) \mid u \in N(v)\}+1 & v \in S \\ c(v) & v \notin S .\end{cases}
$$

Now set $S=A_{1}$ and $G=K G(m, n)$ in (प). The term $\sum_{(u, v) \in I}\left|c^{\prime}(u)-c^{\prime}(v)\right|$ can be calculated exactly, when we use coloring $c^{\prime}$ for $K G(m, n)$. With replacement in ( $(\mathbb{Q})$, we have

$$
\sum D(K G(m, n)) \leq \sum D(K G(m-1, n))+\left(\begin{array}{c}
m-n-1 \\
n-1
\end{array}\right)\left[\left(\begin{array}{c}
m \\
n+1
\end{array}\right)-\frac{n-1}{n+1}\left(\begin{array}{c}
2 n-1 \\
n
\end{array}\right)\right] .
$$




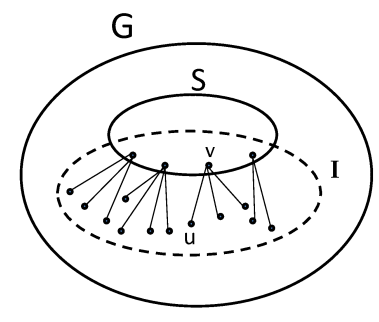

Figure $3 . S$ is an arbitrary independent set of $G$ and $I$ is the set of all edges between $S$ and $G \backslash S$.

By turning $m$ to $m-1$ in (미) after $m-2 n$ iterations, the following inequality would be obtained.

$$
\begin{aligned}
\sum D(K G(m, n)) \leq & \frac{m+1}{2 n+1}\left(\begin{array}{c}
m-n-1 \\
n-1
\end{array}\right)\left(\begin{array}{c}
m \\
n+1
\end{array}\right)-\frac{n-1}{n+1}\left(\begin{array}{c}
2 n-1 \\
n
\end{array}\right)\left[\left(\begin{array}{c}
m-n \\
n
\end{array}\right)-1\right] \\
& -\left(\begin{array}{c}
2 n \\
n+1
\end{array}\right)+\sum D(K G(2 n, n)) \\
& =\frac{m+1}{2 n+1}\left(\begin{array}{c}
m-n-1 \\
n-1
\end{array}\right)\left(\begin{array}{c}
m \\
n+1
\end{array}\right)-\frac{n-1}{n+1}\left(\begin{array}{c}
2 n-1 \\
n
\end{array}\right)\left[\left(\begin{array}{c}
m-n \\
n
\end{array}\right)-1\right] \\
& -\left(\begin{array}{c}
2 n \\
n+1
\end{array}\right)+\frac{1}{2}\left(\begin{array}{c}
2 n \\
n
\end{array}\right) .
\end{aligned}
$$

The term $\frac{1}{2}\left(\begin{array}{c}2 n \\ n\end{array}\right)-\left(\begin{array}{c}2 n \\ n+1\end{array}\right)$ is non-positive for $n \in \mathbb{N}$, since

$$
\begin{aligned}
\frac{1}{2}\left(\begin{array}{c}
2 n \\
n
\end{array}\right)-\left(\begin{array}{c}
2 n \\
n+1
\end{array}\right) & =\frac{(2 n) !}{2 \times n ! n !}-\frac{(2 n) !}{(n-1) !(n+1) !}=\frac{(2 n) !(n+1)}{2 \times n !(n+1) !}-\frac{(2 n) !(2 n)}{2 \times(n) !(n+1) !} \\
& =\frac{(2 n) !(1-n)}{2 \times(n) !(n+1) !} \leq 0
\end{aligned}
$$

and so,

$$
\sum D(K G(m, n)) \leq \frac{m+1}{2 n+1}\left(\begin{array}{c}
m-n-1 \\
n-1
\end{array}\right)\left(\begin{array}{c}
m \\
n+1
\end{array}\right)
$$

Moreover since $K G(m, n)$ is $\left(\begin{array}{c}m-n \\ n\end{array}\right)$-regular graph

$$
\sum S(K G(m, n)) \leq\left(\begin{array}{c}
m-n \\
n
\end{array}\right) \sum_{c} K G(m, n) .
$$

For recent proper coloring $c$

$$
\begin{aligned}
\sum_{c} K G(m, n) & =\left|A_{1}\right|+2\left|A_{2}\right|+\ldots+(m-2 n+1)\left|A_{m-2 n+1}\right|+(m-2 n+2)\left|B_{m-2 n+2}\right| \\
& =\left(\begin{array}{c}
m \\
n
\end{array}\right)\left(\frac{m+1}{n+1}-\frac{n-1}{2 n+2} \frac{\left(\begin{array}{c}
2 n \\
n
\end{array}\right)}{\left(\begin{array}{c}
m \\
n
\end{array}\right)}\right),
\end{aligned}
$$


which is may be the exact value of $\sum K G(m, n)$ [प] . Therefore inequality ([2) implies that

$$
\sum S(K G(m, n)) \leq\left(\begin{array}{c}
m-n \\
n
\end{array}\right)\left(\begin{array}{c}
m \\
n
\end{array}\right)\left(\frac{m+1}{n+1}-\frac{n-1}{2 n+2} \frac{\left(\begin{array}{c}
2 n \\
n
\end{array}\right)}{\left(\begin{array}{c}
m \\
n
\end{array}\right)}\right) .
$$

\section{NON-HOMOMORPHISM THEOREMS}

One of the significant problems in graph theory is graph homomorphism which is relevant to many concepts in this context. The following theorems may be considered as a non-homomorphism theorems which provide two necessary conditions for the existence of homomorphism from a graph $G$ to an edge transitive graph $H$.

Theorem 4.1. Assume that $f: G \longrightarrow H$ is a homomorphism where $H$ is an edge transitive graph. We have

$$
\frac{\Sigma D(G)}{|E(G)|} \leq \frac{\Sigma D(H)}{|E(H)|}
$$

Proof. Assume that $\operatorname{Aut}(H)=\left\{\sigma_{1}, \ldots, \sigma_{n}\right\}$. Let $G_{1}, G_{2}, \ldots G_{n}$ be $n$ isomorphic vertex disjoint copies of $G$ and set $\widetilde{G}=\bigcup_{i=1}^{n} G_{i}$. Consider $\bar{f}: \widetilde{G} \longrightarrow H$ such that the restriction of $\bar{f}$ to $G_{i}$ is $\sigma_{i} o f$, i.e. $\bar{f}_{G_{i}}=\sigma_{i} o f$. It is obvious that $\bar{f}$ is a homomorphism which for any edge $e=a b \in E(H)$, we have $\left|\bar{f}^{-1}(e)\right|=\frac{n|E(G)|}{|E(H)|}$.

Suppose that $c$ is a coloring of $H$ such that $\Sigma_{c} D(H)=\Sigma D(H)$. Naturally, this coloring implies a coloring $\bar{c}$ on the vertices of $\widetilde{G}$. Therefore,

$$
\Sigma_{\bar{c}} D(\widetilde{G})=\frac{n|E(G)|}{|E(H)|} \Sigma_{c} D(H) .
$$

Thus, there is an $i$ such that the restriction of $\bar{c}$ to $G_{i}$ is a coloring $c^{\prime}$ such that

$$
\Sigma_{c^{\prime}} D(G) \leq \frac{1}{n} \Sigma_{\bar{c}} D(\widetilde{G})=\frac{|E(G)|}{|E(H)|} \Sigma_{c} D(H)
$$

On the other hand we know $\Sigma D(G) \leq \Sigma_{c^{\prime}} D(G)$ and so $\Sigma D(G) \leq \frac{|E(G)|}{|E(H)|} \Sigma_{c} D(H)$ which completes the proof.

By the same argument as in proof of theorem (4.]) the next theorem can be proved.

Theorem 4.2. Assume that $f: G \longrightarrow H$ is a homomorphism where $H$ is an edge transitive graph. We have

$$
\frac{\Sigma S(G)}{|E(G)|} \leq \frac{\Sigma S(H)}{|E(H)|}
$$

Inequalities ([4]) and (포) are two necessary conditions for the existence of homomorphism from a graph $G$ to an edge transitive graph $H$.

Corollary 4.3. There is no homomorphism from Petersen graph to $C_{5}$. 
Proof. Note that $C_{5}$ is an edge transitive graph and $\sum D\left(C_{5}\right)=6, \sum S\left(C_{5}\right)=18$. Also, $\sum D(P)=18, \sum S(P)=57$. Accordingly, the inequality (15.5) does not hold but inequality (피) holds. Therefore according to Theorem (4.2) there is no homomorphism from Petersen graph to $C_{5}$.

\section{Some Results And Applications}

The fractional chromatic number of a graph $G$, denoted by $\chi_{f}(G)$, is the infimum of the ratio $\frac{m}{n}$ such that there is a homomorphism from $G$ to $K G(m, n)$. As shown in [22], the infimum can be replaced by minimum. This definition shows that the Kneser graphs play the same central role in fractional graph coloring as the complete graph in graph coloring. It is obvious that $\chi_{f}(G) \leq \chi(G)$.

Theorem 5.1. For every graph $G$ the following inequality holds

$$
\sum D(G)<\chi_{f}(G)|E(G)|
$$

Proof. Assume that $\chi_{f}(G)=\frac{m}{n}$ and $\operatorname{Hom}(G, K G(m, n)) \neq \emptyset$. According to $|E(K G(m, n))|=$ $\frac{\left(\begin{array}{c}m-n \\ n\end{array}\right)\left(\begin{array}{c}m \\ n\end{array}\right)}{2}$ and inequality (ㅁ) and also Theorem 4.1 , it can be concluded that

$$
\begin{aligned}
\sum D(G) & \leq \sum D(K(m, n)) \frac{|E(G)|}{|E(K G(m, n))|} \\
& \leq \frac{2 n(m+1)}{(n+1)(2 n+1)}|E(G)| \leq \frac{m+1}{n+1}|E(G)|<\frac{m}{n}|E(G)|=\chi_{f}(G)|E(G)|
\end{aligned}
$$

$\square$

Theorem 5.2. For every graph $G, \sum S(G)<2 \chi_{f}(G)|E(G)|$.

Proof. The proof is stated on inequality ([03) and an argument similar to Theorem (5.]).

Now assume that $G$ is an edge transitive graph and $\omega(G)$ is the size of maximum clique of $G$. Since there exists a homomorphism from $K_{\omega(G)}$ to $G$, Theorem 4.7 implies, $\sum D(G) \geq \frac{\omega(G)+1}{3}|E(G)|$ and similarly, $\sum S(G) \geq(\omega(G)+1)|E(G)|$.

The next theorem presents another upper bound for the edge-difference chromatic sum of graphs in terms of their chromatic number.

Theorem 5.3. For every graph $G$ we have
a) $\sum D(G) \leq \frac{(\chi(G)+1)}{3}|E(G)|$
b) $\sum S(G) \leq(\chi(G)-1)|E(G)|$ 
Proof. Because of similarity, we just prove the first conclusion. Assume that $f: G \longrightarrow K_{n}$ is a homomorphism where $\chi(G)=n$. By Theorem 4 .], we have $\Sigma D(G) \leq \frac{\mid E(G)}{\left|E\left(K_{n}\right)\right|} \Sigma D\left(K_{n}\right)$. One can easily check that $\left|\Sigma D\left(K_{n}\right)\right|=\frac{n(n-1)(n+1)}{6}$ and therefore, we have

$$
\Sigma D(G) \leq|E(G)| \frac{(n+1)}{3}
$$

as desired.

\section{Conclusions}

In this paper the edge-difference chromatic sum, $\sum D(G)$ and edge-sum chromatic sum, $\sum S(G)$ as two concepts of coloring problem are introduced. According to these concepts the necessary conditions for the existence homomorphism between two graphs are given. As a result of this condition, we presented some lower bounds for $\chi_{f}(G)$. Moreover these bounds will be better if $\sum D(K G(m, n))$ and $\sum S(K G(m, n))$ are accurately calculated.

\section{AcKnowledgments}

The authors wish to sincerely thank the referees for several useful comments.

\section{REFERENCES}

[1] M. Alishahi and A. Taherkhani, A Note on Chromatic Sum, To appear, Ars Combinatoria. Available at arXiv:0802.1936.

[2] E. Kubicka, The chromatic sum of a graph, Western Michigan University, 1989, Michigan.

[3] K. J. Supowit, Finding a Maximum Planar Subset of a Set of Nets in a Channel, Trans. Comp.-Aided Des. Integ. Cir. Sys., 6 (1) (2006) 93-94.

[4] M.R. Garey, and D.S. Johnson, Computers and Intractability; A Guide to the Theory of NP-Completeness, W. H. Freeman \& Co., New York, NY, USA, 1990.

[5] K. E. Stecke, Design, planning, scheduling, and control problems of flexible manufacturing systems, Annals of Operations Research, 3 (1) (1985), 1-12.

[6] C. H. Papadimitriou and K. Steiglitz, Combinatorial Optimization: Algorithms and Complexity, PrenticeHall, Inc., Upper Saddle River, NJ, USA, 1982.

[7] E. K. Burke and B. McCollum and A. Meisels and S. Petrovic and R. Qu, A graph-based hyper heuristic for educational timetabling problems, European Journal of Operational Research, 176 (1) (2007), 177 - 192.

[8] D.H. Smith and S. Hurley and S.U. Thiel, Improving heuristics for the frequency assignment problem, European Journal of Operational Research, 107, (1) (1998)76-86.

[9] D. de Werra and Ch. Eisenbeis and S. Lelait and B. Marmol, On a graph theoretical model for cyclic register allocation, Discrete Applied Mathematics, 93 (2-3) (1999) 191-203.

[10] M. Gamache and A. Hertz and J. O. Ouellet, A Graph Coloring Model for a Feasibility Problem in Monthly Crew Scheduling with Preferential Bidding, Comput. Oper. Res., 34 (8) (2007) 2384-2395. 
[11] C. A. Glass, Bag rationalisation for a food manufacturer, Journal of the Operational Research Society, 53 (5) (2002) 544-551.

[12] P. Erdos and E. Kubicka and A. J. Schwenk, Graphs that require many colors to achieve their chromatic sum, In Proceedings of the Twentieth South-eastern Conference on Combinatorics Graph Theory and Computing, 71 (1990) 17-28.

[13] Yu. Li, C. Lucet, A. Moukrim, K. Sghiouer, Greedy Algorithms for the Minimum Sum Coloring Problem, (2007) Sousse, Tunisia.

[14] H. Hajiabolhassan and M.L. Mehrabadi and R. Tusserkani, Minimal coloring and strength of graphs, Discrete Mathematics, 215 (1) (2000) 265 - 270.

[15] H. Hajiabolhassan and M. L. Mehrabadi and R. Tusserkani, Tabular Graphs and Chromatic Sum, Discrete Mathematics, 304 (1-3) (2005) 11-22.

[16] L. G. Kroon, A. Sen, H. Deng and A. Roy, The Optimal Cost Chromatic Partition problem for trees and interval graphs, Graph-Theoretic Concepts in Computer Science: 22nd International Workshop (1997) 279-292.

[17] T. Jiang and D. B. West, Coloring of Trees with Minimum Sum of Colors, J. Graph Theory, 32 (4) (1999) $354-358$.

[18] E. Kubicka A. J. and Schwenk, An Introduction to Chromatic Sums, Proceedings of the 17th Conference on ACM Annual Computer Science Conference, (1989) 39-45.

[19] , C. Thomassen and P. Erdos and Y. Alavi and P. J. Malde and A. J. Schwenk, Tight bounds on the chromatic sum of a connected graph, Journal of Graph Theory, 13 (3) (1989) 353-357.

[20] U. Benlic and J. K. Hao, A Study of Breakout Local Search for the Minimum Sum Coloring Problem, Simulated Evolution and Learning: 9th International Conference, (2012) 128-137.

[21] M. Malafiejski, Sum coloring of graphs, Contemporary Mathematics: Graph Colorings, (2004) 55-65.

[22] E..R. Scheinerman and D.H. Ullman, Fractional graph theory: A rational approach to the theory of graphs, John Wiley \& Sons Inc., New York, 1997.

[23] P. Hell and J. Nesatril, Graphs and homomorphisms, Oxford University Press, 2004.

\section{S. Rahimi Sharbaf}

School of Mathematical Science, Shahrood University of Technology, Shahrood, Iran.

srahimi@shahroodut.ac.ir

\section{Kh. Erfani}

School of Mathematical Science, Shahrood University of Technology, Shahrood, Iran.

erfani@shahroodut.ac.ir 